\title{
Blue-domed cyst with optic nerve compression
}

\author{
M I T CHEL L D. B UR N B A U M, JOH N W. HAR B ISON, \\ JOH N B. SELHORST, AND HAROLDF. YOUNG
}

From the Department of Neurology and Division of Neurosurgery of The Department of Surgery, Medical College of Virginia, Richmond, Virginia, USA

SUMMARY A patient presenting with persistent headache of vascular quality and an intermittently progressive chiasmal syndrome had a blue-domed haemorrhagic cyst beneath the right optic nerve and underwent surgical drainage with good results. As prompt surgical intervention may provide significant return of visual function, detailed diagnostic evaluation of similar cases is recommended.

Isolated suprasellar cysts are rare and may be clinically and radiologically indistinguishable from more common neoplasms of the parasellar region. In this report, we present a patient whose clinical picture was caused by recurrent haemorrhage into such a suprasellar cyst. The clinical and histopathological features of cystic suprasellar mass lesions are reviewed.

\section{Case report}

A previously healthy 43 year old motel manager woke up on 6 October 1973 with a severe right frontotemporal throbbing headache. A headache of varying intensity continued for five months and, when most severe, was accompanied by an illusion of bright, multicoloured, pulsatile bursts of light in his right eye. In November 1973, the patient noted dim vision in his right eye, and became aware of persistent drowsiness. He could not define clearly the onset of either problem.

When examined in March 1974, the right eye visual acuity was $20 / 30$. There was a right afferent pupillary defect, colour vision was markedly impaired, and the right optic disc was pale. A right inferior altitudinal defect was demonstrated by tangent screen examination. The left eye was normal as was the remaining neurological examination.

Routine skull radiography demonstrated normal size and configuration of the sella turcica. The EEG, brain scan, spinal fluid examination, and a right carotid arteriogram were normal. An ex-

Address for reprint requests: Dr John W. Harbison, Department of Neurology, Box 698, MCV Station, Medical College of Virginia, Richmond, Virginia, 23298, USA.

Accepted 26 May 1978 planation of the patient's right optic neuropathy was not established.

Severe headaches continued despite a variety of analgesics. In May 1974, repeated visual field examination showed further depression of the peripheral isopters in the right eye, and the patient was placed empirically on prednisone, $60 \mathrm{mg}$ daily. The headaches stopped within a few days. Visual function improved marginally during the next 15 months on gradually tapering doses of prednisone.

In September 1975, prednisone was discontinued. One month later, the patient experienced sudden, severe right fronto-orbital pain accompanied by abrupt loss of vision in the right eye. The visual acuity was reduced to counting fingers. A dense central and inferior altitudinal scotoma was demonstrated in the right visual field. The left visual field showed depression in the superior temporal area which extended to the vertical midline (Fig. 1).

A CAT scan disclosed a small, midline suprasellar density that did not enhance with injection of contrast medium (Fig. 2). Optic canal polytomograms were normal and, at pneumoencephalography, air was seen in the chiasmatic cistern on the lateral view. Pneumopolytomography of the sella turcica, however, demonstrated a mass in the region of the optic chiasm, indenting the third ventricle (Fig. 3). The spinal fluid was xanthochromic, and contained more than 5000 red blood cells per $\mathrm{mm}^{3}$. A complete endocrine evaluation was normal.

A right frontal craniotomy was performed. Retraction of the right frontal lobe disclosed a small, $10 \mathrm{~mm}$ diameter, dark blue cyst underlying the right optic nerve (Fig. 4). The mass was 


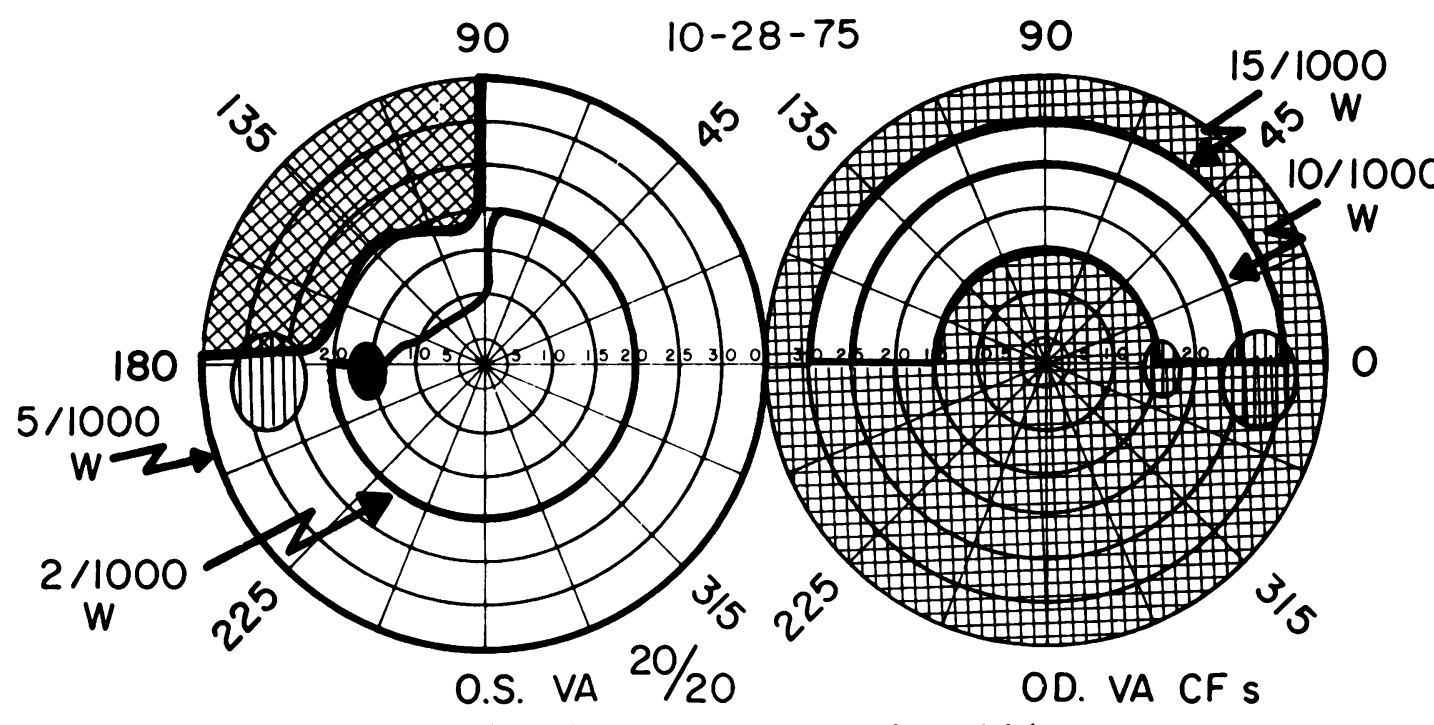

Fig. 1 Tangent screen visual field of October 1975 demonstrating chiasmal defect.

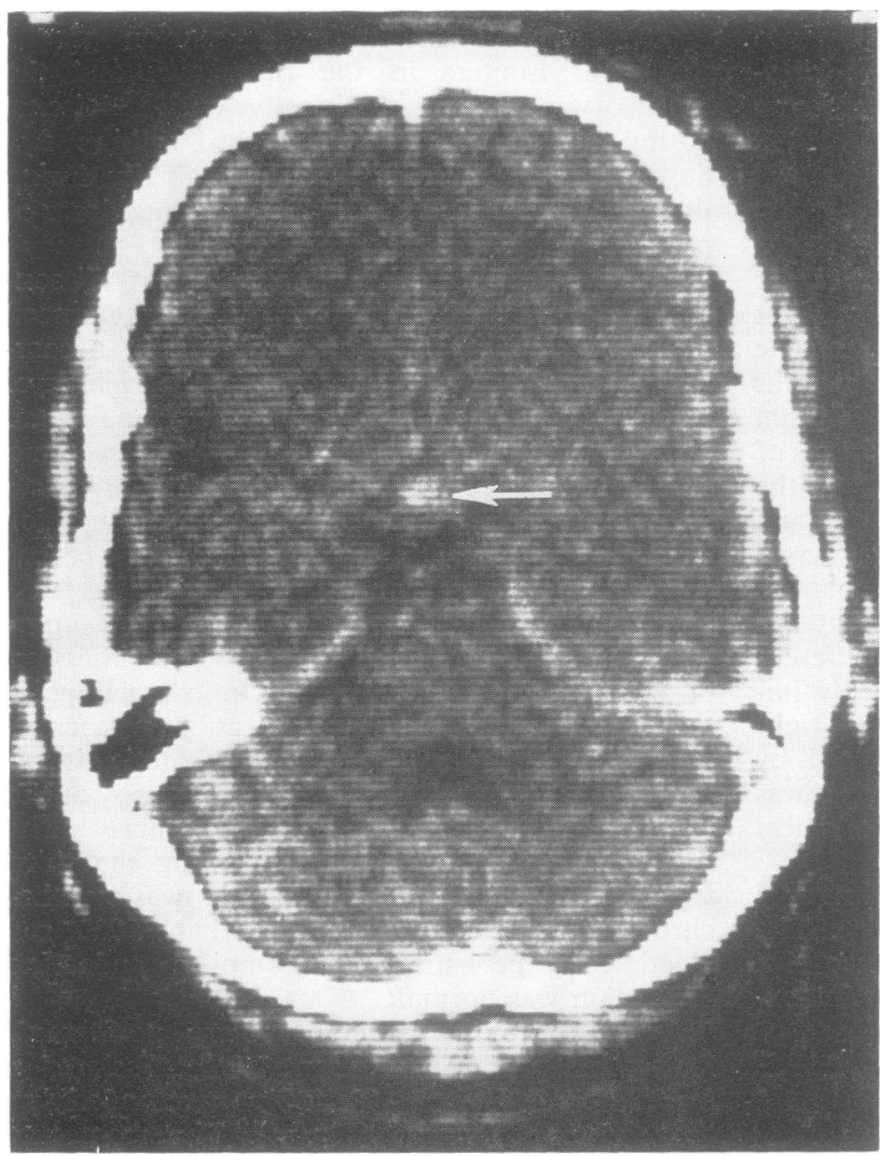

Fig. 2 Unenhanced computerised tomography shows a small midline suprasellar area of increased density (arrow). 


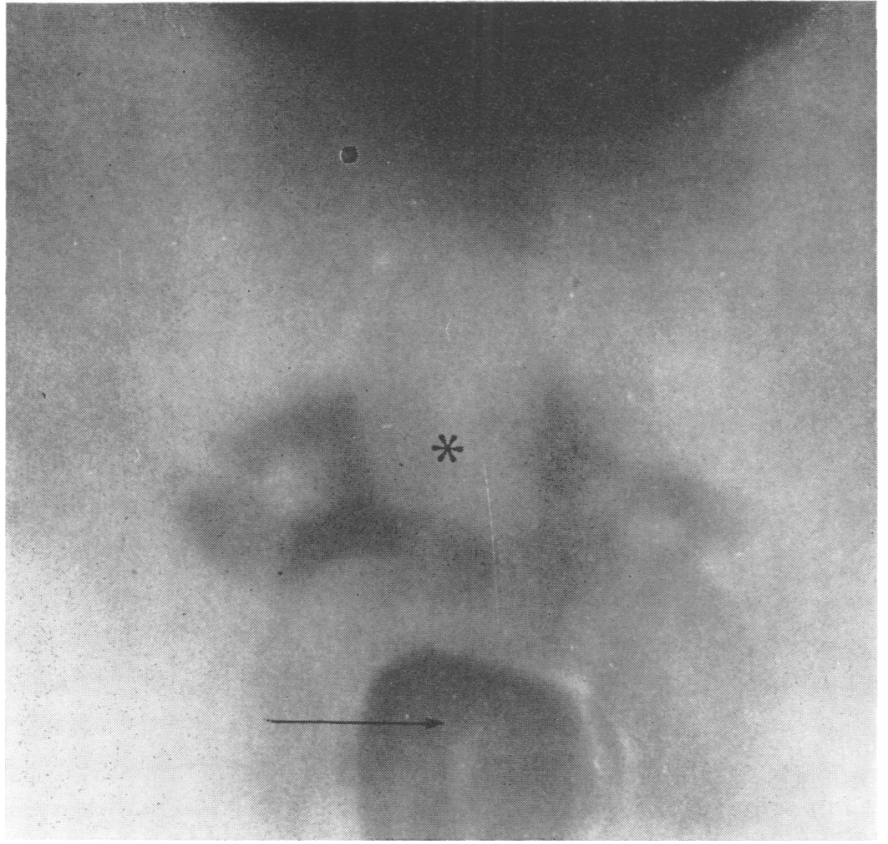

Fig. 3 Coronal pneumopolytomogram, section through the sella turcica shows a midline suprasellar density in the region of the optic chiasm (asterisk). Arrow indicates position of the sphenoidal sinus.

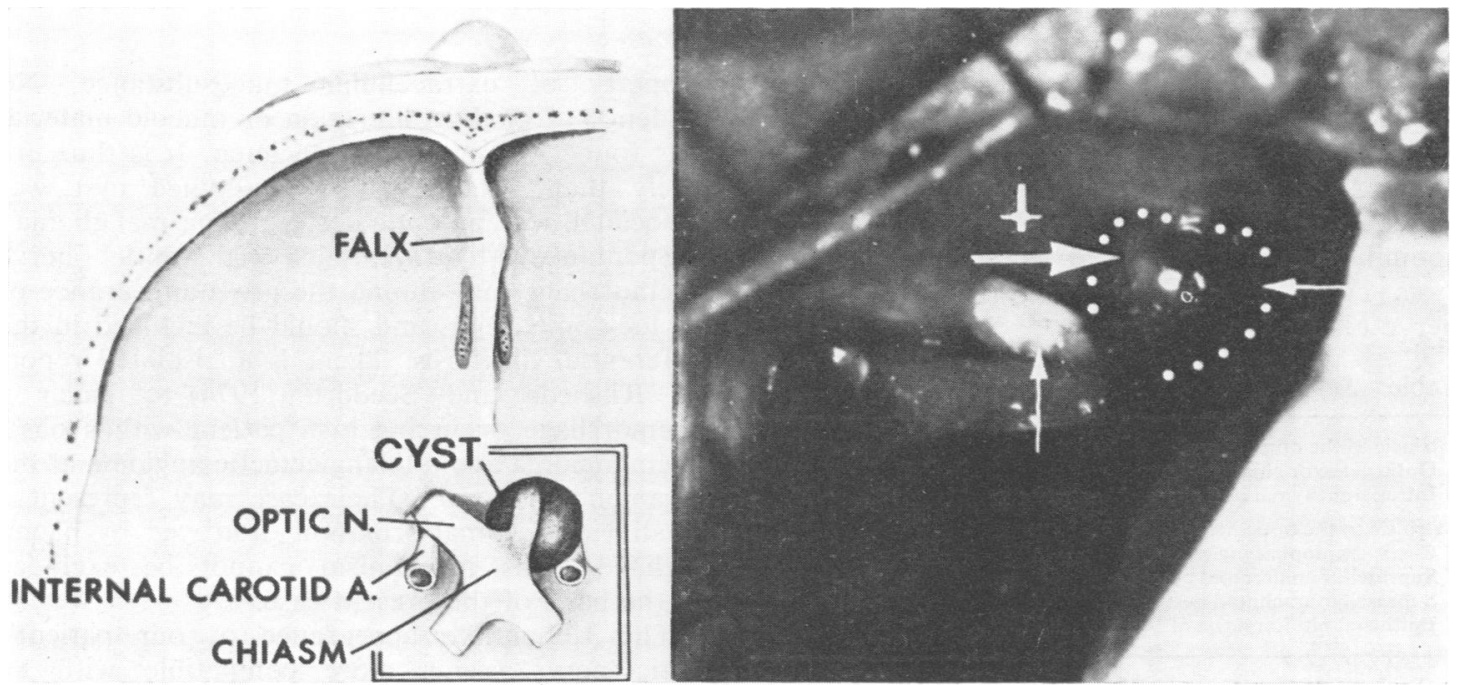

Fig. 4 Schematic representation of blue-domed cyst underlying a markedly thinned right optic nerve.

tapped using a 25 gauge needle, and $0.5 \mathrm{ml}$ of dark blue, viscous fluid was drained. After drainage, the right optic nerve was examined and appeared markedly thinned but otherwise normal. A small specimen of cyst wall was taken from the undersurface of the nerve.

The cyst wall was composed of dense, fibrocollagenous tissue, and contained an abundance of haemosiderin pigment. The cyst lumen was filled with organising haematoma, and was lined by flat, meningothelial-like cells (Fig. 5). Despite a careful review of several sections, no columnar or cuboidal lining cells were identified.

Six months after surgery, visual acuity was $20 / 50$ in the right eye, and tangent screen visual field examination demonstrated a marked reduction of the central scotoma and expansion of the peripheral isopter in the superior field. 


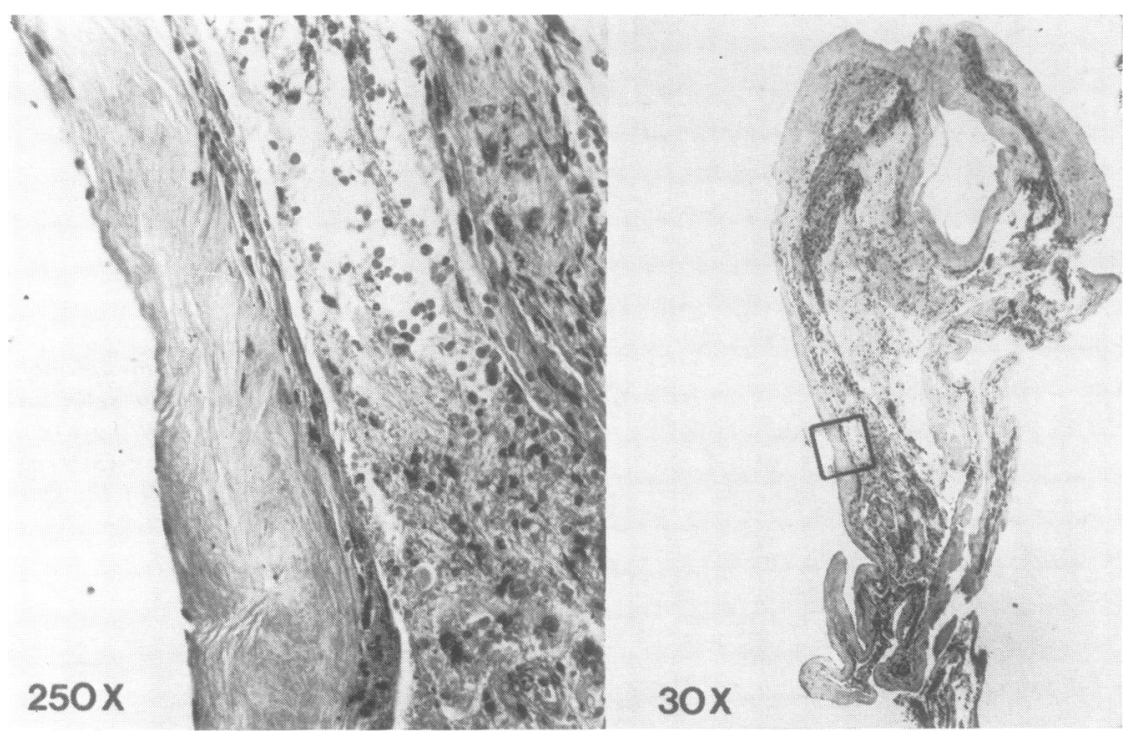

Fig. $5 H$ and $E$ histological section of cyst. Right photograph shows complete cyst in cross-section, original magnification $\times 30$. Left photograph is enlargement of cyst wall taken from area of rectangle in photograph of the complete cyst, original magnification $\times 250$. It demonstrates fibrocollagenous wall with flat meningothelial-like cell lining.

\section{Discussion}

Despite a careful review of all histological sections of the cyst wall, the precise nature of our patient's haemorrhagic cyst remains unclear. A compilation of diagnostic possibilities includes cysts both intrinsic and extrinsic to the optic nerve (Table).

\section{Table Suprasellar cysts: differential diagnosis}

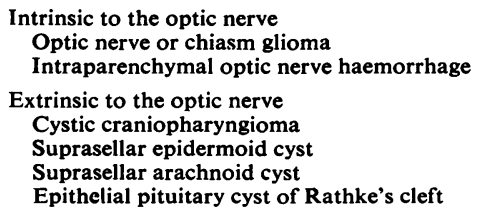

Although Schneider et al. (1970) discuss a "blue, dome-shaped infarct" on the surface of an optic nerve glioma, no similar example is reported elsewhere. The blue-domed cyst in our patient was clearly beneath the optic nerve, elevating and thinning it, and did not resemble an infarct. Abrupt decline in visual function, as in our patient, occurs infrequently with gliomas. Anderson and Spencer (1970) present such a patient and attribute the visual loss to rapid enlargement of tumour caused by the hydrophilic property of extracellular mucosubstance. No evidence of glial proliferation or mucoid materialo $z$ was found in our biopsy specimen. It is thus unlikely that our patient's blue-domed cyst was associated with an optic nerve or chiasmal glioma.

Spontaneous intraparenchymal optic nerve haemorrhage may mimic the gross appearance of a blue-domed cyst, and should be included in the differential diagnosis. There is an isolated report by Riishede and Seedorff (1974) of such a haemorrhage occurring in a patient with a nasal haemangioma and a telangiectactic angioma at the foramen of Monro. Their case may represent a small vascular malformation destroyed by haemorrhage. This mechanism cannot be excluded as the basis of the present case.

The ribbon-like appearance of our patient's optic nerve seems more compatible with an extrinsic cyst than with an intrinsic neoplasm or cyst. Cystic craniopharyngiomas and epidermoid cysts are commonly suprasellar and are distinguished by their content of cholesterol crystals and keratin, as well as by their stratified squamous cell lining. These pathological features were not present in our case, and thus neither are considered likely in our patient.

In the suprasellar region arachnoid cysts and epithelial cysts of Rathke's cleft origin must also be recognised and distinguished (Ringel and Bailey, 
1972). Although generally intrasellar, epithelial cysts may be partially or entirely suprasellar, and are reported to be blue-domed (Fager and Carter, 1966). They are consistently filled with a mucoid substance, but the cyst wall may be highly vascular, and intraluminal haemorrhage is reported (Weber et al., 1970). In the presence of recurrent haemorrhage, the typical cuboidal or columnar lining cells of a Rathke's cleft cyst may be flattened or destroyed, making histological differentiation from an arachnoid cyst difficult. The histological appearance of our patient's cyst most closely resembled that of an arachnoid cyst in that its lumen was lined by meningothelial-like cells. In a case similar to ours Barlow (1935) described an isolated suprasellar arachnoid cyst compressing the optic nerves and chiasm. It did not, however, have a haemorrhagic character.

We believe our patient's cyst was either an arachnoid cyst or an epithelial cyst of Rathke's cleft. Its deep blue colour probably represents recurrent haemorrhage into this pre-existing cyst. Pathological demonstration of old and recent blood, the stuttering clinical course, and spinal fluid xanthochromia are consistent with recurrent haemorrhage.

Suprasellar cysts, though rare, are surgically treatable causes of optic nerve and chiasm compression. Recovery of visual function is dependent upon prompt, persistent diagnostic evaluation and surgical drainage. The small size of these cysts, however, may make diagnosis by routine neuroradiological techniques difficult. Although computerised axial tomography may be helpful in the rare instance of intraluminal haemorrhage, pneumoencephalography with AP polytomography appears to be the diagnostic procedure of choice when a small suprasellar mass is suspected.

\section{References}

Anderson, D. R., and Spencer, W. H. (1970). Ultrastructural and histochemical observations of optic nerve gliomas. Archives of Ophthalmology, 83, 324 335.

Barlow, A. (1935). Suprasellar arachnoid cyst. Archives of Ophthalmology, 14, 53-60.

Fager, C. A., and Carter, H. (1966). Intrasellar epithelial cysts. Journal of Neurosurgery, 23, 77-81.

Riishede, J., and Seedorff, H. H. (1974). Spontaneous hematoma of the optic chiasma. Acta Ophthalmologica, 52, 317-322.

Ringel, S. P., and Bailey, O. T. (1972). Rathke's cleft cyst. Journal of Neurology, Neurosurgery, and Psychiatry, 35, 693-697.

Schneider, R. C., Kriss, F. C., and Falls, H. F. (1970). Prechiasmal infarction associated with intrachiasmal and suprasellar tumours. Journal of Neurosurgery, 32, 197-208.

Weber, E. L., Vogel, F. S., and Odom, G. L. (1970). Cysts of the sella turcica. Journal of Neurosurgery, $33,48-53$. 